\title{
Monstre intensifieur en français
}

\author{
Gilles Corminboeuf ${ }^{1, *}$ et Mathieu Avanzi ${ }^{2}$ \\ ${ }^{1}$ Université de Fribourg, Avenue de Beauregard 13, 1700 Fribourg, Suisse \\ ${ }^{2}$ STIH, Sorbonne Université, 5 rue Victor Cousin, 75005 Paris, France
}

\begin{abstract}
Résumé. Le but de cet article est de présenter les résultats d'une recherche relative à l'usage de monstre antéposé à un lexème qu'il modifie (il fait monstre chaud). Dans un premier temps, nous montrons comment les données récoltées dans le cadre d'un projet faisant appel aux sciences participatives nous permettent de localiser avec précision la forme en Suisse romande et dans quelques localités de France voisine. Dans un second temps, nous exploitons le corpus OFROM et les données récoltées dans d'autres sources pour cerner les contraintes distributionnelles qui pèsent sur l'utilisation de ce morphème en français. Nous terminons sur les propriétés sémantiques qui invitent à décrire monstre comme un morphème intensifieur.
\end{abstract}

\begin{abstract}
The aim of this paper is to present the results of a research dealing with the word monstre ("monster"), placed before a lexical unit or a phrase that it modifies (as in il fait monstre chaud, "it is very hot"). First, we use crowdsourced data to draw with precision the boundaries of the area where this singular use of monstre is attested, i.e. in French-speaking Switzerland and in some neighboring localities of France. Next, thanks to the data extracted from the OFROM database and from other resources, we examine the distributional properties of monstre in French. Third, we describe the semantic properties of the intensificator monstre.
\end{abstract}

\section{Introduction}

Cet article décrit l'usage d'un régionalisme grammatical du français, usité en Suisse romande et dans les départements français limitrophes - à savoir le morphème monstre employé en tant qu'intensifieur d'une unité lexicale contiguë (les exemples sous 1 proviennent de scripteurs suisses sur Twitter; ils ont été retranscrits comme tels) :

(1) (a) le monstre fric qu'ils doivent choper en ingame transactions pfiouuuu

(b) Du coup j'enlève le couvercle et y'a la monstre fumée qui sort et le fond qui est tout tout noir

(c) On a la monstre neige

(d)@Daavv01viens rider a Verbier les canonniers font monstre neige du coup pas inquiet du tout

(e) Les salles de sport en Suisse elles coutent monstre cher, mais comme dans ma tête je suis riche je vais leur donner leur nonante francs an patate

* gilles.corminboeuf@unifr.ch 
(f) Ce soir on est monstre chaud, nos joueurs \#R6S participent à une (a)Glory4GamersFR

(g) En plus je suis déjà monstre crevée de mon weekend alors je vais pas tenir sans un peu de repos, sans mon temps libre :(

(h) Mais oui. "Monstre bonne journée," mérite aussi

(i) Je me suis monstre pris la tête toute seule et je suis habillée comme un sac putain

(j) Il monstre neige !

L'usage de monstre comme épithète est attesté dans les variétés dites "de référence » du français, comme le confirme une recherche dans le corpus ESLO, qui a permis d'extraire 19 occurrences du lemme monstre. Parmi ces dix-neuf occurrences, treize sont des emplois substantivaux (p. ex. c'est devenu un monstre), sept au singulier et six au pluriel. L'autre emploi observé, adjectival, est attesté à six reprises :

(2) (a) $[\ldots]$ à cause de cet embouteillage monstre

(b) une boîte de nuit où il y a de la fumée + un bruit monstre

(c) moi elle m'a fait une impression monstre

(d) il est arrivé à une pagaille monstre

(e) ça coûte un fric monstre hein l'embellissement d'une ville hein

(f) c'est vrai que ça coûte quand même un fric euh assez monstre

Dans (2), monstre fonctionne comme une épithète postposée et correspond à la description de l'emploi adjectival donnée dans un paragraphe de l'entrée monstre du TLFi :

Emploi adj., fam. [En parlant d'une chose concr. ou abstr.] Exceptionnel par la quantité, la taille, l'intensité. Synon. colossal, phénoménal, prodigieux. Un banquet monstre ; un succès monstre. La fameuse collection de couteaux, allant depuis le catalan monstre jusqu'à un eustache grand comme le petit ongle de sa petite main (GONCOURT, Journal, 1858, p.529). Chadourne nous prépare un breakfast monstre café au lait, oeufs, confiture, papaye, bananes (GIDE, Retour Tchad, 1928, p.939). Courtial en était outré de ce culot monstre! (CÉLINE, Mort à crédit, 1936, p.558). (Le Trésor de la langue française, TLFi)

Trois éléments sont notables dans cette définition : d'une part, cet emploi est tenu pour «familier » par le TLFi; d'autre part monstre peut modifier un N concret (banquet) ou abstrait (culot); et enfin, il est fait mention d'une composante quantitative-intensive (« exceptionnel par la quantité [...] l'intensité. Synon. colossal, phénoménal, prodigieux»).

Ce qui constitue le caractère régional des emplois réunis sous (1), c'est la position antéposée de monstre (monstre fric, monstre fumée), et le fait qu'il puisse modifier une autre catégorie qu'un substantif - par exemple un adjectif (monstre cher, monstre chaud), un prédicat verbal (je suis déjà monstre crevée, je me suis monstre pris la tête) ou un adverbe (infra, §4.2.3).

L'emploi singulier de monstre illustré en (1) n'a pas été consigné dans les dictionnaires et autres glossaires consacrés au français régional de Suisse romande et de France voisine. Il n'est mentionné ni dans le Dictionnaire suisse romand (Thibault 2004), ni dans le Dictionnaire des régionalismes de France (Rézeau 2001), ni dans le Dictionnaire des régionalismes de Rhône-Alpes (Fréchet 2015), pour ne citer que les principaux. Les seules attestations que nous avons trouvées sont contenues dans des ouvrages datant du début des années 1980. On trouve la première dans un document de travail dactylographié (Tuaillon \& Rézeau 1983 : 127), et qui le localise en Haute-Savoie (v. l'acception adverbiale) :

MONSTRE, adj. et adv. 1. Adj. Acharné, supérieur aux autres. Il est monstre pour faire ses randonnées tout seul en montagne. 2. Adv. Très. Cette bestiole est monstre petite. H.-Sav. 
La seconde figure dans un relevé des particularités lexicales du français parlé en Vallée d'Aoste (Martin $1984:$ 32) :

MONSTRE, adj. « énorme, très grand ». Il avait une monstre cicatrice (S.D., agric. 49, m. Jov.). Voy. FEW VI, 3, 99, monstrum, fr. monstrueux, adj. « ayant une conformation contre nature, excessif (laideur, grosseur ») »; GoD, V. 394. Monstrueux; RoB., IV, 640, $4^{\circ}$ cite monstre employé adject. et fam. Avec le sens de « extraordinaire » dans l'ordre quantitatif. Dans notre tém., il y a en plus antéposition de l'adjectif alors que CH. VAUT., VII, 483 citent monstro, adj., « colossal, prodigieux, extraordinaire » et donnent des exemples où l'adj. est toujours postposé.

Nous verrons comment l'exploitation de données récoltées dans le cadre d'enquêtes en ligne nous permet de préciser les frontières géographiques de l'aire où on peut entendre cet usage de monstre ( $\$ 2)$, et comment les attestations extraites du corpus OFROM et d'autres sources nous permettent de caractériser ses contraintes distributionnelles $(\S \S 3-4)$. Avant de conclure avec les différentes questions que cette étude laisse ouvertes (\$6), nous revenons brièvement sur les propriétés sémantiques de monstre, et justifions ainsi son statut d'intensifieur (§5).

\section{Aire géographique et vitalité : l'apport du crowdsourcing}

Malgré les progrès qu'ont connu les corpus oraux des vingt dernières années (Avanzi, Béguelin \& Diémoz 2016a), l'apport des archives de parole transcrites demeure toujours insuffisant quand il est question de documenter certains faits de langue, qu'il s'agisse de diastratismes (Gadet 2000, 2017) ou de régionalismes, notamment grammaticaux (Avanzi, Béguelin \& Diémoz 2016b : §44). Dans ce contexte, certains chercheurs se sont tournés vers des méthodes alternatives, en mettant à profit les méthodes expérimentales qui ont fait leurs preuves en psycholinguistique (Dagnac \& Thuilier, à par.). D'autres ont exploité le potentiel des réseaux sociaux pour administrer en ligne ce que les premiers spécialistes du français régional ont appelé des " enquêtes de vitalité » (Rézeau 2001). C'est cette seconde option qui a été exploitée dans le cadre du programme de recherche Français de nos Régions. Nous ne reviendrons pas sur les principes méthodologiques qui sous-tendent ces enquêtes, ni sur leurs avantages et leurs limites ${ }^{1}$. Nous nous contenterons de rappeler que depuis le lancement du projet (mai 2015), des dizaines d'enquêtes ont été mises en ligne, et qu'elles ont permis de cartographier avec précision la vitalité et l'aire d'extension de phénomènes diatopiquement marqués, qu'ils relèvent du lexique, de la grammaire ou de la prononciation (Avanzi 2017, 2018, 2019). Bon nombre des réponses aux questions contenues dans ces enquêtes n'ont jamais été dépouillées, et demandent encore à être exploitées. C'est le cas en particulier des intensifieurs en français, domaine que l'on sait particulièrement sensible à la variation (Dostie 2018). Pour cet article, nous nous sommes servis des matériaux de deux enquêtes. La première, ci-après " euro-1 », a été administrée en 2015 ; la seconde, ci-après «euro-4 », a été administrée en 2017. La première enquête comportait une question formulée de la façon suivante : "L'Apple Watch, la montre connectée d'Apple, vient juste de sortir. Vous aimeriez bien l'avoir, mais vous hésitez à l'acheter, car...». La question était accompagnée de la photo d'une Apple Watch ${ }^{\circledR}$, ainsi que des choix de réponses suivants : (i) elle est très chère, (ii) elle est méga chère, (iii) elle est super chère, (iv) elle est trop chère, (v) elle est grave chère, (vi) elle est monstre chère et (vii) autre (précisez). Compte tenu du grand nombre de suggestions reçues lors de la première enquête, la question a été reposée dans le $4^{\mathrm{e}}$ sondage de la série. Elle était alors formulée ainsi : «En français, il existe des dizaines de façons de dire que quelque chose est 'trop' ou 'super' bien. Dans la liste ci-dessous, cochez le ou les mots que vous utilisez dans vos conversations entre amis ou en famille pour remplacer le mot manquant dans la phrase 'c'est [...] bien' ». Elle était suivie de la liste de propositions que voici : (i) cher, (ii) fin, (iii) 
fort, (iv) gavé, (v) grave, (vi) hart ${ }^{2}$, (vii) hyper, (viii) méga, (ix) monstre, (x) pire, (xi) plein, (xii) rien, (xiii) tarpin, (xiv) très, (xv) trop, (xvi) vrai, (xvii) vachement, (xviii) drôlement et (xix) autre (précisez).

\subsection{Participants}

Dans l'enquête euro-1, plus de 10.700 internautes ont répondu à la question relative aux intensifieurs. Dans l'enquête euro-4, ils étaient un peu moins de 8.300. Les tables 1. et 2. rendent compte des principales caractéristiques de ces internautes en fonction de leur pays d'origine, de leur âge et de leur genre :

Tableau 1. Participants à l'enquête euro-1, avec leur région d'origine, nombre, pourcentage par rapport au total, sexe et âge.

\begin{tabular}{|l|l|l|l|l|l|l|l|}
\hline \multirow{2}{*}{ pays } & \multirow{5}{*}{ N } & \multicolumn{2}{|l|}{ Sexe } & \multicolumn{3}{l|}{ Âge (années) } \\
\cline { 3 - 8 } & & $\mathbf{H}$ & $\mathbf{F}$ & moyenne & min. & max. & écart-type \\
\hline Belgique & 435 & 189 & 246 & 34,9 & 14 & 84 & 14,5 \\
\hline France & 8.409 & 3.075 & 5.334 & 34,8 & 11 & 91 & 13,2 \\
\hline Suisse & 1.887 & 752 & 1135 & 38,8 & 12 & 94 & 15,9 \\
\hline Total & $\mathbf{1 0 . 7 3 1}$ & $\mathbf{4 . 0 1 6}$ & $\mathbf{6 . 7 1 5}$ & $\mathbf{3 5 , 8}$ & $\mathbf{1 1}$ & $\mathbf{9 4}$ & $\mathbf{1 3 , 8}$ \\
\hline
\end{tabular}

Tableau 2. Participants à l'enquête euro-4, avec leur région d'origine, nombre, pourcentage par rapport au total, sexe et âge.

\begin{tabular}{|l|l|l|l|l|l|l|l|}
\hline \multirow{2}{*}{ pays } & \multirow{2}{*}{ N } & \multicolumn{2}{|l|}{ Sexe } & \multicolumn{3}{l|}{ Âge (années) } \\
\cline { 3 - 8 } & & $\mathbf{H}$ & $\mathbf{F}$ & moyenne & min. & max. & écart-type \\
\hline Belgique & 189 & 98 & 91 & 34,2 & 10 & 76 & 15,3 \\
\hline France & 7.890 & 2.995 & 4.895 & 30,9 & 10 & 89 & 12,6 \\
\hline Suisse & 179 & 87 & 92 & 37,4 & 10 & 77 & 16,7 \\
\hline Total & $\mathbf{8 . 2 5 8}$ & $\mathbf{3 . 1 8 0}$ & $\mathbf{5 . 0 7 8}$ & $\mathbf{3 1 , 2}$ & $\mathbf{1 0}$ & $\mathbf{8 9}$ & $\mathbf{1 2 , 9}$ \\
\hline
\end{tabular}

\subsection{Cartographie}

Les données relatives à la localisation des participants nous ont permis de calculer leur nombre pour chaque arrondissement de Belgique, de France et de district en Suisse. Nous avons alors pu, pour chaque possibilité de réponse proposée dans le questionnaire, calculer le pourcentage d'utilisation de telle ou telle forme d'intensifieur. Nous avons reporté les résultats sur un fond de carte, en faisant varier la couleur des points proportionnellement aux valeurs des pourcentages obtenus. À partir de ces valeurs, nous avons utilisé la méthode du krigeage (Gratton 2002) pour obtenir une surface lisse et continue ${ }^{3}$.

2.2.1 Les données de la première enquête ont permis de rendre compte du fait que l'emploi régional de monstre n'est guère répandu en dehors des frontières de la Suisse romande : tous points confondus, les pourcentages y avoisinent $30 \%$ d'emploi déclaré, contre à peine $1 \%$ pour la Belgique et pour la France. 
Si à présent on zoome sur la partie Centre-Est de la francophonie d'Europe, on peut formuler deux remarques, l'une relative à la Suisse, l'autre à la France.

D'abord, on peut voir que la vitalité de monstre intensif régional n'est pas la même d'un bout à l'autre de la Suisse romande : les pourcentages les plus hauts, qui se situent autour de 50\%, sont atteints dans le canton de Fribourg (54\% dans le district de la Sarine, 50\% dans les districts du Lac, de la Glâne et de Veveyse) et la partie dite "savoyarde » du Valais (Saint-Maurice : 57\%, Entremont : 50\% et Martigny : 48\%). Dans le canton de Vaud, les pourcentages avoisinent $40 \%$ dans les districts les plus à l'ouest (Jura-Nord vaudois et Lavaux-Oron : $42 \% ; 38 \%$ dans le Gros-de-Vaud et 37\% dans le district de la Riviera-Pays-d'Enhaut). Ailleurs en Suisse, les pourcentages sont en moyenne de $25 \%$ sans descendre en dessous de la barre de $15 \%$, à part dans les districts du Locle, de Bienne et de Porrentruy (12,5\% en moyenne).

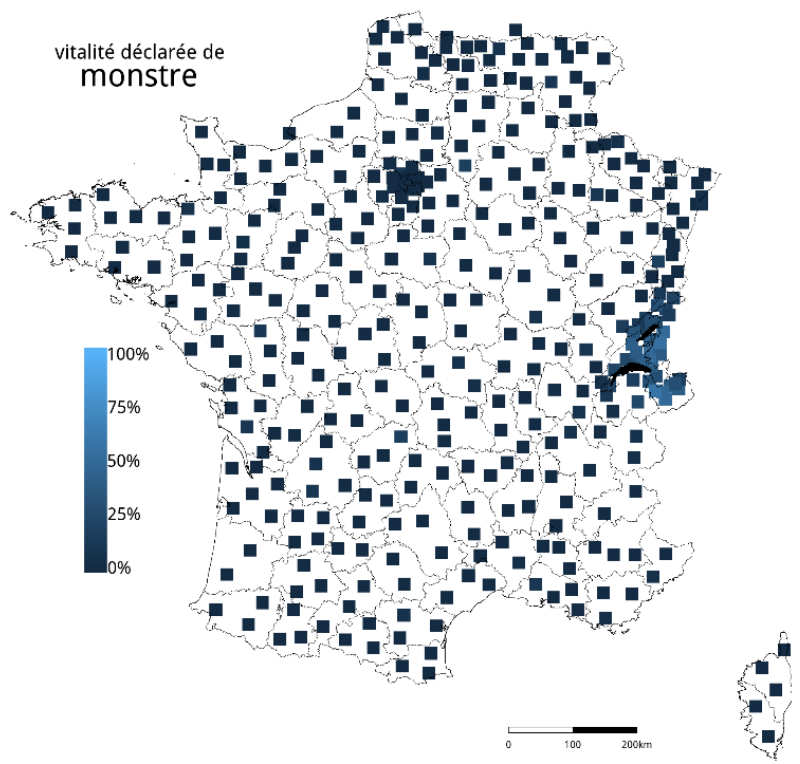

Fig. 1. Pourcentage d'usage déclaré de la forme monstre dans la phrase elle est monstre chère, enquête euro-1. Les points signalent les positions d'arrondissements en Belgique et en France, de districts en Suisse. 


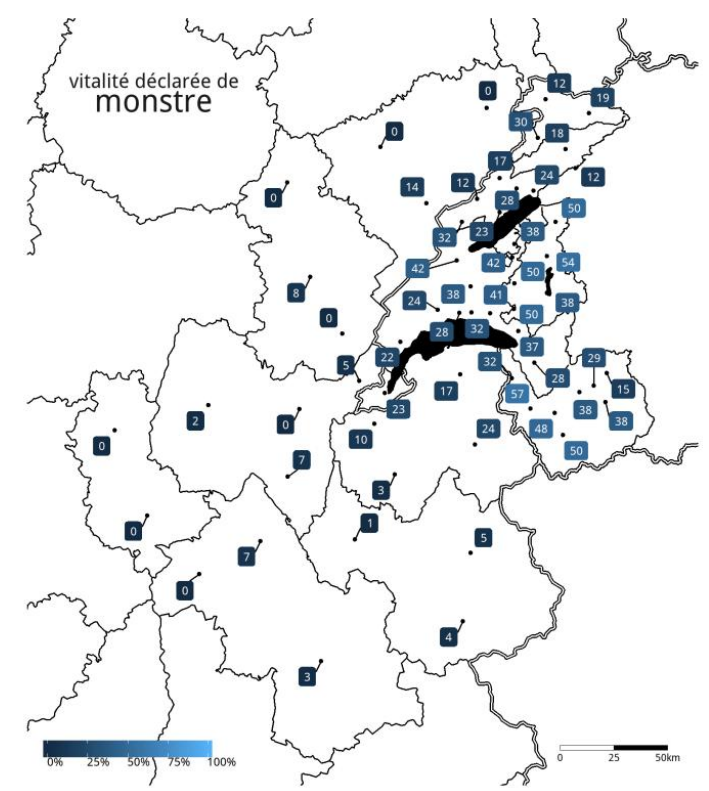

Fig. 2. Pourcentage d'usage déclaré de la forme monstre dans la phrase elle est monstre chère, enquête euro-1. Les points signalent les positions d'arrondissements en France et de districts en Suisse.

Ensuite, du côté de la France voisine, les résultats sont majoritairement nuls ou inférieurs à $10 \%$, à part dans les arrondissements les plus proches de la frontière : $14 \%$ à Pontarlier (Doubs), 24\% à Bonneville et $17 \%$ à Thonon-les-Bains (tous deux en Haute-Savoie).

2.2.2 Bien que le nombre de participants ne soit pas comparable dans l'enquête euro- $4^{4}$, les résultats de la Fig. 3. ne sont pas contradictoires avec ceux qui sont reportés sur la Fig. 2. Les pourcentages les plus hauts (100\%) sont obtenus dans les districts de la Glâne et de la Broye (canton de Fribourg), de Saint-Maurice (Valais) et du Val-de-Travers (Neuchâtel). La présence de valeurs nulles en Suisse s'explique par le manque de participants.

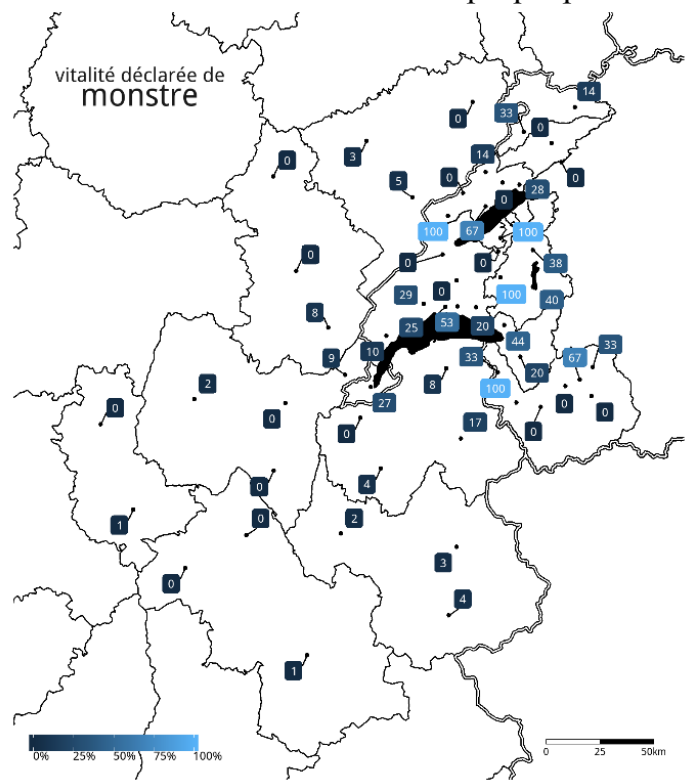

Fig. 3. Pourcentage d'usage déclaré de la forme monstre dans la phrase : c'est monstre bien, enquête euro-4. Les points signalent les positions d'arrondissements en France et de districts en Suisse. 
En France voisine, un pourcentage de 10\% est dépassé seulement dans l'arrondissement de Hauteville, en Haute-Savoie.

\subsection{Statistiques}

Les données récoltées dans l'enquête euro-1 étant relativement nombreuses, nous avons pu tester si l'usage de monstre intensif antéposé était conditionné par l'âge des internautes. L'idée était de vérifier si l'usage de monstre était typique de ce qu'on appelle « les parlers jeunes ». Pour coller au mieux à la réalité des faits, nous avons isolé les points du réseau auxquels étaient associés des pourcentages supérieurs à 10\%. Nous avons ainsi retenu l'ensemble des participants de Suisse romande $(\mathrm{N}=1.887)$ et les internautes ayant déclaré avoir passé la plus grande partie de leur jeunesse dans les arrondissements de Pontarlier $(\mathrm{N}=28)$, Saint-Julien-en-Genevois $(\mathrm{N}=20)$ et Thonon-les-Bains $(\mathrm{N}=24)$, soit 1.959 internautes au total.

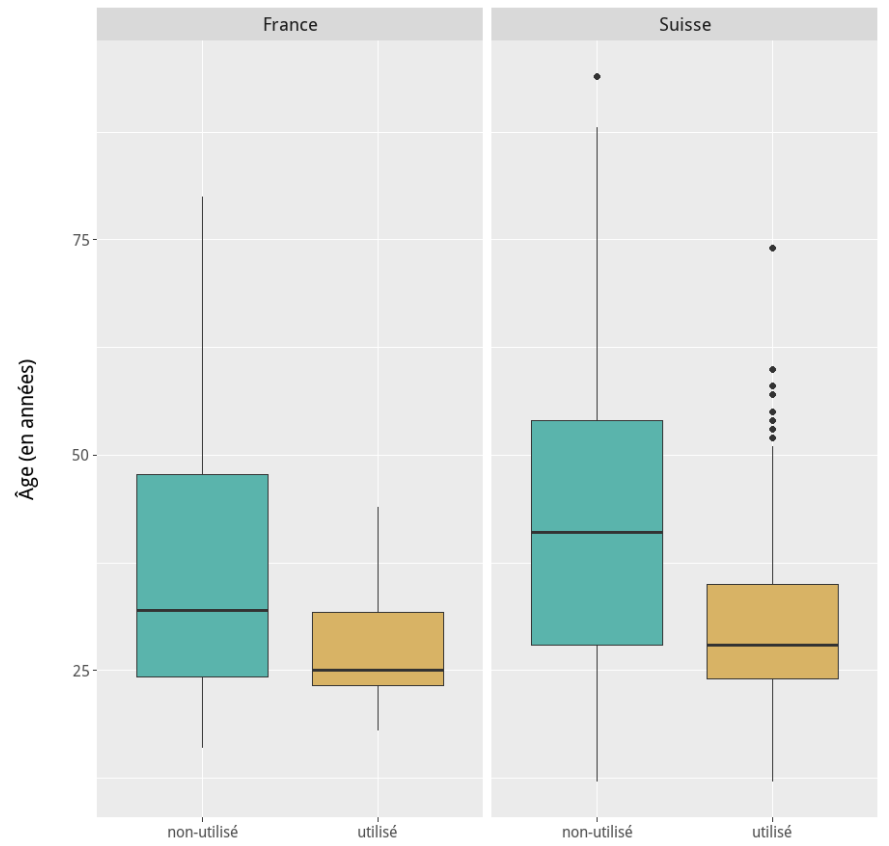

Fig. 4. Distribution de l'âge des participants de Suisse romande et de France voisine qui ont déclaré utiliser ou non la forme monstre dans la phrase elle est monstre chère, enquête euro-1.

Un modèle linéaire avec comme variable dépendante l'âge des participants, et l'interaction entre l'utilisation de monstre et le pays d'origine des participants a montré que la distribution que l'on observe sur la Fig. 4. n'était pas due au hasard $(\mathrm{F}=1,1956 ; \mathrm{p}<$ 0,001). En d'autres termes, les participants qui utilisent monstre comme intensifieur sont significativement plus jeunes (en moyenne : 28 ans en France, 30 ans en Suisse) que ceux qui ont déclaré ne pas utiliser cette forme (en moyenne : 36 ans en France, 42 ans en Suisse $)^{5}$.

\section{Les contextes distributionnels dans OFROM}

Les résultats des enquêtes Français de nos Régions ont permis de tracer les frontières de l'aire d'usage de monstre intensifieur, et de montrer qu'il s'agissait plutôt d'une forme que l'on retrouve dans la bouche des plus jeunes (bien que nos données indiquent qu'on 
l'entend aussi chez les locuteurs plus âgés : dans 4b infra, la locutrice a 63 ans et dans 5a, 51 ans). Pour aller plus loin dans la description et établir les contextes distributionnels de cet emploi, nous avons cherché l'ensemble des occurrences dans le corpus OfROM (Avanzi, Béguelin \& Diémoz 2016b), qui totalise plus d'un million de mots.

Dans le corpus OfROM, on compte 35 occurrences du lemme monstre. Nous laissons de côté l'emploi substantival ( 3 exemples au singulier, 3 exemples au pluriel). Le statut très minoritaire de cet emploi dans OFROM est cependant instructif, si on le compare avec les 13 occurrences substantivales sur les 19 au total issues du corpus orléanais ESLO (v. supra ex. 2). Les 29 exemples non substantivaux ressortissent à quatre contextes distributionnels distincts (infra, §§3.1-3.4). Une seule de ces occurrences (ex. 3, infra) correspond à la définition du TLFi (supra, §1). Les 28 occurrences restantes de monstre n'appartiennent pas au « français de référence » : ce sont des occurrences qui sont non substantivales et non postposées à un nom. Ces formes sont produites par 15 locuteurs différents $(7$ femmes, 8 hommes). La plupart des locuteurs sont étudiants, mais on trouve également une secrétaire, un spécialiste marketing, un ambulancier, une maîtresse enfantine et un constructeur métallique.

Il est notable que 8 des occurrences recueillies apparaissent dans la même interaction, dont l'enregistrement dure 20 minutes, entre un homme et une femme qui produisent chacun 4 occurrences de monstre. L'homme réalise par ailleurs 3 des 5 occurrences où monstre apparaît dans un SN défini, du type elle avait le monstre espoir (§3.2). Même si 4 des 5 occurrences de SN défini sont produites par deux locuteurs valaisans, il serait hasardeux de conclure à une micro-variation régionale: en l'absence de données supplémentaires, on considérera provisoirement qu'il n'y a pas non plus de variation d'un canton suisse à l'autre. Quant à l'âge des 15 locuteurs, 10 ont moins de 25 ans, 2 ont entre 25 et 30 ans et 3 entre 44 et 63 ans (dont deux femmes). Si elle n'est pas réservée aux "parlers jeunes », la forme monstre est néanmoins sinon un indicateur, du moins un indice parmi d'autres de «parler jeune », ce qui est cohérent avec ce que l'on a observé plus haut (v. §2.3). Précisons enfin que pour tout locuteur de Suisse romande, cet usage de monstre est toutefois parfaitement intelligible - même s'il ne produit pas lui-même la forme en question.

\subsection{Contexte 1 : monstre épithète postposée}

Une seule occurrence présente une épithète postposée :

(3) à chaque match ça doit leur faire un: un fric monstre quoi

On peut se demander si fric monstre ne fonctionne pas à la manière d'une collocation, ce qui expliquerait la postposition. Notons toutefois que l'antéposition est également attestée (1a, supra) et que dans cette position monstre est graduable (assez, dans $2 \mathrm{f}$, supra), ce qui interroge précisément l'hypothèse collocationnelle.

\subsection{Contexte 2 : monstre épithète antéposée}

16 occurrences contiennent une forme monstre(s) en position d'épithète antéposée. Nous distinguons les onze SN indéfinis (4) des cinq SN définis (5) :

(4) (a) pis il s'envoie une monstre pétée + dans la gueule + pis il continue à marcher

(b) on peut pas y aller à pied l'hiver parce qu'y a une euh: monstre montée qui est un peu raide mais c'est quoi à cinq minutes de marche même pas

(c) mais c'est chiant déjà que hier y avait un monstre orage + c'est abusé

(d) purée j'ai un monstre bol 
(e) L1 : ils sont ils ont quoi ils ont une maison?

L2 : ils ont une monstre baraque

(f) putain eh j'ai vraiment foutu une monstre merde euh

(g) c'est une tour avec + une monstre pub BMW dessus

(h) j'en connais des gens qui se sont retrouvés tout seuls dans les moments difficiles + eh ben ça fait des dégâts quoi + ça fait des ça fait des monstres dégâts euh

(i) ceux qui sont vraiment très atteints + s'envoient des monstres + coups de poing dans la tête [à propos du syndrome de Gilles de la Tourette]

(j) mais c'était + bon hein il faisait des des monstres trucs la bonne soupe

(k) on a été manger à la Buat Tower + euh + c'est une tour euh parce qu'y a des des monstres euh des des gratte-ciels euh à Bangkok ${ }^{6}$

Dans les extraits (4), monstre(s) se laisse gloser par l'épithète énorme. Mais la glose serait plus exacte en écrivant énorme comme certains scripteurs sur le web énoorme, énooorme, énaurrme, formes phonographiques (Mahrer 2017) suggérant une intensité maximale au moyen d'un mime de la prosodie (allongement et proéminence accentuelle). À témoin le tracé intonatif de (4a), généré à l'aide du logiciel Praat (Boersma \& Weeninck 2019) :

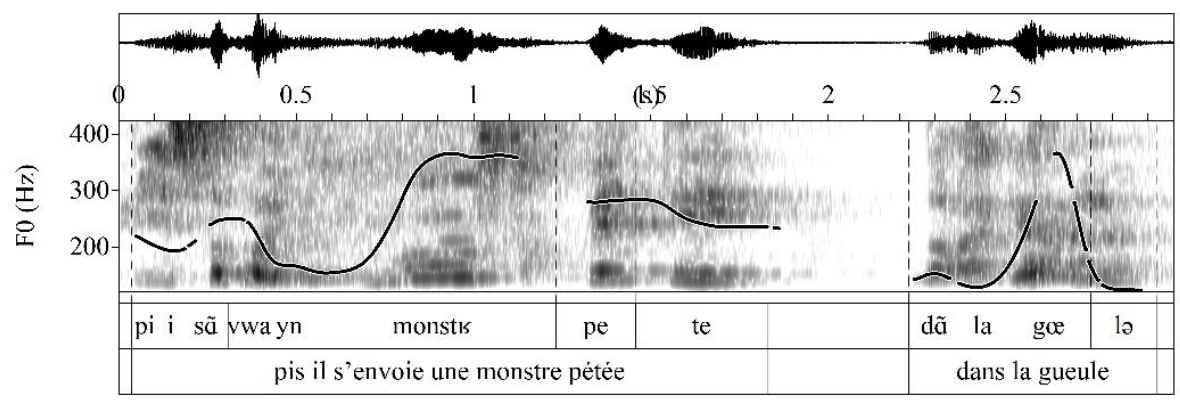

Fig. 5. Représentation intonative stylisée de (4a) «pis il s'envoie une monstre pétée + dans la gueule ».

On peut voir sur cet exemple que le mot monstre est assorti d'une montée mélodique remarquable (plus de $12 \mathrm{dt}$ ), aussi importante que celle du contour en cloche dont est assortie la syllabe métrique finale de l'énoncé (dans la gueule). La durée de la syllabe monstre est largement supérieure à celle des autres syllabes de l'énoncé : avec une durée de $685 \mathrm{~ms}$, cette syllabe est trois fois plus longue que les autres (la moyenne des syllabes dans cet énoncé étant de $240 \mathrm{~ms}$ ). On retrouve une configuration intonative similaire sur l'exemple (5a), où l'on observe une montée intonative de près de $10 \mathrm{dt}$ (l'allongement relatif n'est pas si important $-580 \mathrm{~ms}$ contre $334 \mathrm{~ms}$ pour la moyenne totale des syllabes de l'énoncé - mais reste quand même notable) : 


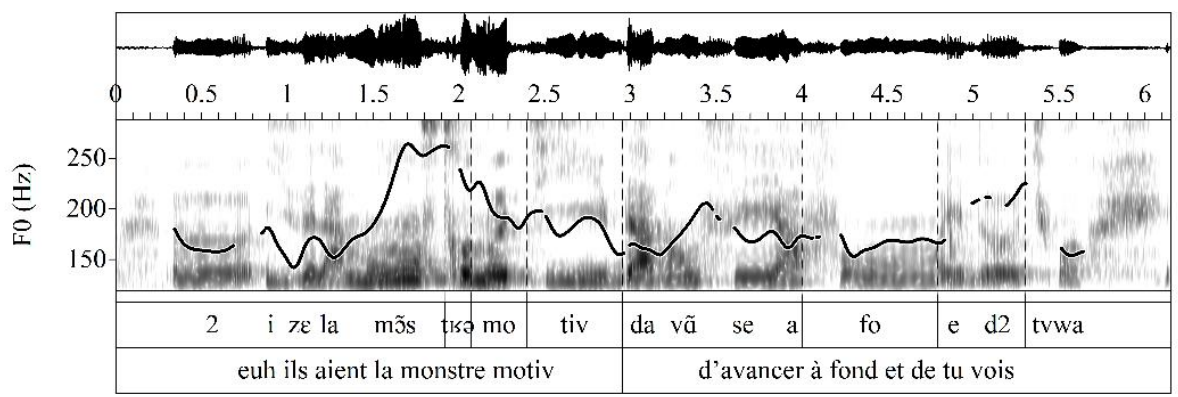

Fig. 6. Représentation intonative stylisée de (5a) « euh ils aient la monstre motiv d'avancer à fond et de tu vois ».

Les occurrences (5) présentent un SN défini :

(5) (a) je crois pas qu'au cycle + euh: ils aient la monstre motiv d'avancer à fond [le « cycle d'orientation » correspond plus ou moins au collège en France]

(b) donc c'est trop marrant parce que t'as le monstre contraste tu sais

(c) elle a le monstre souci pour faire la valise c'est tellement marrant

(d) ouais écoute il aime bien + il a eu un peu de peine au début à s'adapter pis là maintenant il a- +

L2 : ouais

L1 : il a la monstre joie

(e) en plus là e- elle avait le monstre espoir et tout

Le monstre $N$ se glose par « le plus intense $\mathrm{N}$ qui soit» (cf. elle avait le monstre espoir $\rightarrow$ 'elle avait l'espoir le plus intense qui soit'). L'usage du défini présente l'objet-dediscours désigné comme unique en son genre, placé à un degré maximal d'intensité. Le choix du déterminant défini s'explique par le fait qu'il n'y a qu'un seul plus haut degré, ce qui apparente cet emploi au superlatif.

Les occurrences (5) suggèrent une attraction des $\mathrm{N}$ abstraits pour le déterminant défini. Cela dit, il ne s'agit sans doute que d'une tendance, à témoin (6) :

(6) il roulait avec le monstre pick-up (oral, à la volée, canton de Fribourg ; 14.03.2019; à propos d'un nouveau voisin)

Dans (6), le substantif pick-up a le trait [+concret], ce qui montre que la cooccurrence déterminant défini-N concret n'est pas exclue.

Dans (4) et dans (5), des indices confirment la lecture intensive : chiant / abusé dans (4c), vraiment dans (4f), très dans (4i); ̀̀ fond dans (5a), trop dans (5b), tellement dans $(5 \mathrm{c})$.

\subsection{Contexte 3 : monstre intensifieur d'adjectif}

Dans 9 occurrences du corpus OFROM, monstre intensifie un adjectif scalaire. Positionné après le verbe être ou faire, cet adjectif endosse la fonction d'attribut :

(7) (a) ils sont monstre hauts dans le euh dans l'organigramme quoi

(b) c'est monstre cool + tu vas avec qui ?

(c) j'essaie de regarder comment je dois faire le truc quoi + c'est monstre chaud + mais je crois que je tiens le bon bout 
(d) pis après on devait tout faire plein de trucs c'était monstre chaud

(e) mais le problème c'est qu'il va faire monstre froid

(f) mais il fait monstre froid déjà il est en bonnet gants

(g) c'est monstre beau hein la ville

(h) L1 : t'es allé en Thaïlande pis t'es pas allé à la plage ?

L2 : si je suis allé à la plage

L1 : ouais pis c'était pas monstre beau ? ++ euh j'ai toujours entendu dire que les plages en Thaïlande c'était incroyable

(i) je suis pas monstre bien quoi

L'intensifieur monstre peut être glosé par "extrêmement" ${ }^{\text {" }}$. Il modifie également des prédicats qui commutent avec des attributs, comme le SP à la mode ou le SN la classe dans $(8)$ :

(8) (a) Je ne connaissait pas du tout il y a 2 semaines et maintenant je vois que c'est monstre à la mode dans plein de pays et que ça débarque ici aussi. (web, sic, https://bebe.ch)

(b) Et c est monstre la classe d avoir même son propre site pour nous faire découvrir ce que vous vivez... (web, sic, https://maxetsasha.com)

\subsection{Contexte 4 : monstre intensifieur de prédicat verbal}

Dans 3 occurrences du corpus OFROM, monstre se place entre l'auxiliaire et l'auxilié d'une forme verbale au passé composé, à savoir dans une position prototypique pour un adverbe :

(9) (a) il a monstre poussé le gazon

(b) ça a été le bordel depuis le début + ils se sont monstre pris la tête tout le long

(c) il a monstre maillé sur [nom propre] ('il s'est énervé contre lui, il a râlé sur lui')

Dans (9), monstre se glose par un adverbe du type "énormément ${ }^{9}$. Dans ce contexte 4 , le verbe tensé n'est cependant pas forcément à la forme accomplie (infra, §4.2.2 et supra, $1 \mathrm{j})$.

\section{L'apport de données issues d'autres sources}

L'emploi intensif de monstre dans OFROM n'étant attesté que dans une petite trentaine d'occurrences, nous avons recoupé nos observations avec d'autres sources. À défaut d'une étude détaillée, nous nous en tiendrons ici à quelques observations sur l'écrit - qui confirment (§4.1) ou conduisent à affiner nos contextes distributionnels (§4.2).

\subsection{L'écrit comme confirmation}

Les données de presse écrite ${ }^{9}(10 a)$ et (10b) concernent le compte-rendu du même incendie en Gironde : le périodique suisse antépose l'épithète (10a), alors que le périodique français le postpose (10b) :

(10) (a) Le monstre incendie près de Bordeaux d'origine criminelle ? [titre]

France — Le feu a détruit 530 hectares de pinède, nécessitant l'évacuation d'environ 600 personnes. (presse écrite, Le matin, 26.07.2015 ; Saint-Jean-d'Illac)

(b) Incendie monstre en Gironde : 4ème jour de lutte pour les sapeurs-pompiers (presse écrite, www.planet.fr ; 27.07.2015, à propos du même incendie) 
Dans la presse suisse romande, il n'est pas rare d'observer cet emploi de monstre :

(11) (a) $[\ldots]$ elles font désormais partie du club très privé des détenteurs d'une cabine [...] « Nous savions que c'était difficile d'en avoir une. On n'y croyait plus. On se sent monstre Fribourgeoises avec elle !» (presse écrite, La Liberté, 05.07.2018; à propos de la location d'une cabine personnelle à la piscine communale de Fribourg)

(b) Caricature dans la presse écrite (La Liberté, 11 mai 2019 ; à propos de l'arrêt du Cirque Nock pour raisons financières et de la fraude électorale présumée à Genève, qui survient après de nombreuses affaires peu reluisantes qui ont secoué le monde politique genevois)

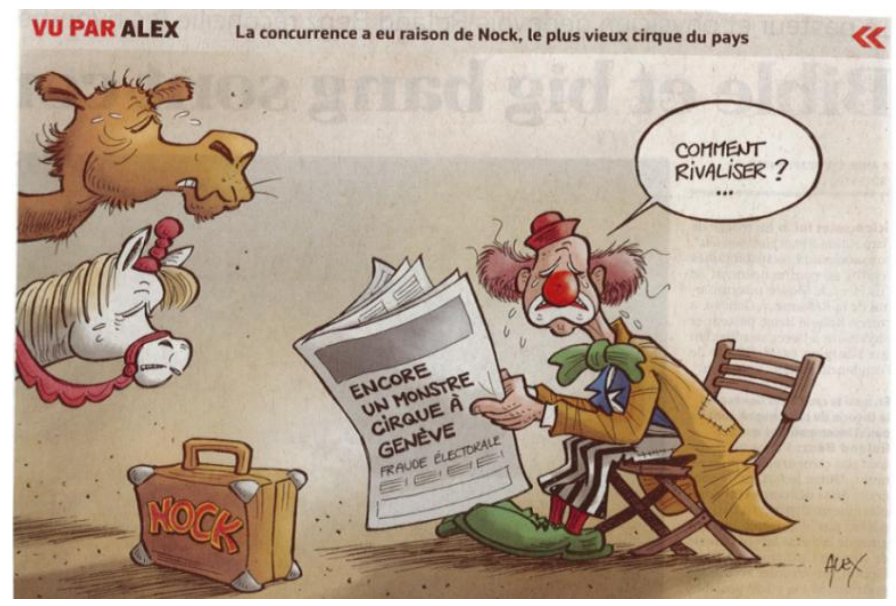

(c) Emission radiophonique (Airs de rien, 01.01.2018, 20h02; https://www.rts.ch/play/radio/airs-de-rien/audio/monstre-cool?id=9182401)

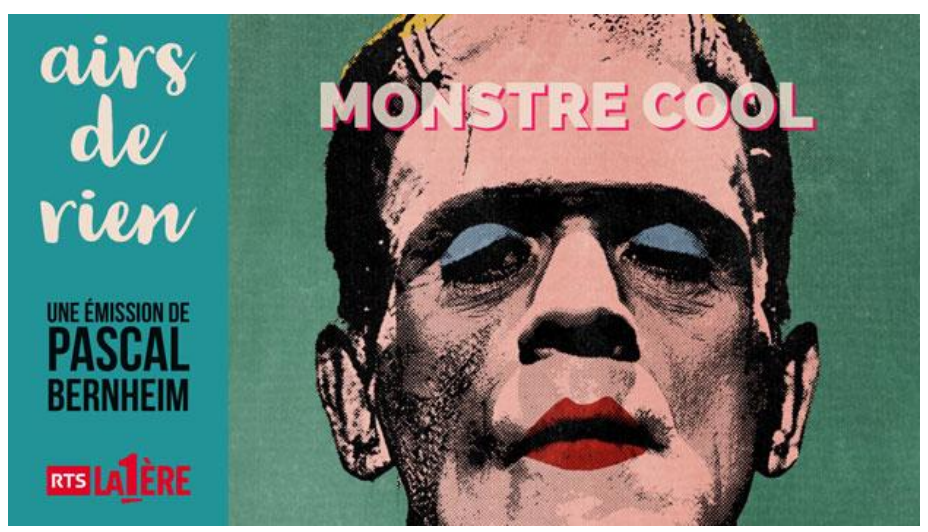

Dans (11a), monstre intensifie l'attribut Fribourgeoises (contexte 3), alors que dans (11b) monstre est épithète antéposée du N cirque (contexte 2). L'expression monstre cool (11c) est ambiguë en français de Suisse romande: monstre est-il un N expansé par l'épithète cool ou un intensifieur de l'épithète cool ? L'usage de la technique sérigraphique warholienne, qui permet de superposer les images de Frankenstein (Boris Karloff) et de Marylin Monroe, entretient ici l'ambiguïté d'analyse.

Le Corpus suisse de sms (sms.linguistik.uzh.ch), fournit les occurrences suivantes :

(12) (a) On prend un monstre apéro au rosé !

(b) coucou, si ça dérange pas trop je vais pas venir diner.... Monstre boulot ..... 
(c) c tjs un monstre plaisir de te voir !

(d) Monstre flippant !

Dans (12a-c), monstre est épithète antéposée (contexte 2) et dans (12d) intensifieur d'adjectif (contexte 3).

\subsection{Affinage des contextes distributionnels}

4.2.1 La prise en compte d'occurrences issues d'autres sources qu'OFROM conduit aux observations suivantes. D'une part, il convient de déterminer un contexte 3bis (cf. supra §3.3.), où monstre intensifieur d'épithète est postposé :

(13) Mais punaise, c'est beau monstre, surtout avec cette peinture. (web, www.gtanf. com)

4.2.2 D'autre part, certaines occurrences conduisent à poser l'existence d'un contexte 4bis (cf. supra, §3.4.), où monstre intensifie un prédicat verbal, mais a posteriori.

(14) (a) il a neigé monstre! (oral, à la volée, 16.01.2019, canton de Fribourg; un adolescent au téléphone dans le train)

(b) on les a plantés y a quatre ans et ils ont poussé monstre tu vois (oral, à la volée, 31.07.2019, canton de Fribourg; à propos des arbustes d'une haie privée)

(c) Jetais meme pas dans les pogos j'etais tout devant et sa poussait monstre (web, sic, https://stramberyfish.skyrock.com ; à propos d'un concert)

Monstre apparaît après une forme verbale accomplie (cf. contexte 4 supra, et 14a-b) ou inaccomplie (14c). La postposition de l'intensifieur après un SV (14) ou après une séquence \{verbe + attribut (13) n'est pas (encore ?) attestée dans OFROM.

4.2.3 Enfin, les faits empiriques (15) nous conduisent à poser un contexte $\mathbf{5}$ où monstre est incident à un adverbe lui-même déjà intensifieur (notamment grave et beaucoup) :

(15) (a) Mec cis : sois là en soutien et si t'es relou gaffe-toi, on est monstre beaucoup ! (web, https://cerclelabreche.wordpress.com; à propos d'une marche féministe ; cis = 'cisgenre')

(b) j'ai déjà testé ta mousse de mascarpone, je crois bien que je vais récidiver avec cette recette qui a juste l'air de déchirer monstre grave (web, www.beaualalouche. com)

(c) il pleuvait monstre des cordes (web, http://forum-auto.caradisiac.com)

Dans (15c), monstre intensifie le SN lui-même marqueur de haut degré des cordes, qui commute avec un adverbe.

Les exemples ci-dessus suggèrent que monstre acquiert de nouveaux contextes : à droite d'un attribut (13), d'un SV (14) et incident à un adverbe (15) ${ }^{10}$.

\section{Sémantique}

5.1 Anscombre \& Tamba (2013:3), qui soulignent le flou terminologique et catégoriel que présentent les «phénomènes intensifs », montrent que ceux-ci sont rendus linguistiquement aussi bien au niveau lexical (préfixes de haut degré, adverbes d'intensité) qu'au niveau sémantico-syntaxique (exclamatives), prosodique (intonation emphatique, allongement vocalique) ou encore énonciatif et argumentatif (réduplication, saillance). Notre objet d'étude fait intervenir une marque lexicale (monstre), une opposition morpho-syntaxique (SN défini vs indéfini, §3.2) et un profil prosodique localement singulier (§3.2). De plus, monstre peut être dupliqué (infra, ex. 17c). 
Étudiant la catégorie sémantique de l'intensité, Gaatone (2008 : 2495, 2498) rappelle le consensus apparent selon lequel les quantifieurs - qui modifient un substantif ou un verbe, c'est-à-dire des référents quantifiables - sont distingués des intensifieurs - qui portent sur un adjectif ou un adverbe, c'est-à-dire des référents intensifiables. Certaines unités ne fonctionnent que dans l'une des deux catégories, alors que d'autres peuvent opérer à la fois comme quantifieur et comme intensifieur. Kleiber $(2013: 66)$ montre ainsi que dans Paul mange beaucoup à midi, c'est la quantité de nourriture qui est ciblée, alors que dans Marie admire beaucoup Paul il est question d'intensité (i.e. de 'quantité' d'admiration). De même (ibid., 64-67), prototypiquement, lorsque la quantité porte sur des objets ou des événements, la lecture est quantitative : Paul a beaucoup dormi. Lorsque la quantité porte sur des propriétés ou des états, c'est l'intensité qui est en jeu : Paul est très triste / grand (cf. 'Paul est triste à un haut degré'). Dostie résume l'opposition comme suit :

Le quantifieur signale le nombre d'entités $X$ (dans le cas du nom) ou d'événements $X$ (dans celui du verbe) ou, encore, leur importance quantitative (p.ex. beaucoup de chagrin / peu d'idées). [...] L'intensifieur indique la « quantité de qualité » (Kleiber 2007b : 251) associée à une propriété $\mathrm{X}$ quelconque, c'est-à-dire qu'il agit comme une sorte d'instrument qui donne la mesure des traits (définitoires) de ce X, qu'il en précise l'importance sous l'angle de la «grandeur » (petite, moyenne, grande). Par conséquent, si l'on dit de Lucie qu'elle est très intelligente, cela signifie qu'elle possède en grande quantité les attributs qui sont ceux que l'on relie normalement à l'intelligence. (Dostie $2018: 23$ )

Les occurrences d'OFROM suggèrent que monstre fonctionne essentiellement comme un intensifieur. Dans les contextes 3 et 5 , cela est évident, monstre étant incident à une catégorie intensifiable (adjectif et adverbe). Qu'en est-il des contextes 2 et 4 ? Dans le contexte (2), la lecture quantitative apparaît beaucoup moins commune que la lecture intensive (voir les exemples 4 et 5, supra) - même si la catégorie modifiée (substantif et prédicat verbal) orienterait vers la lecture quantitative. Voyons toutefois les extraits suivants :

(a) le monstre fric qu'ils doivent choper (= 1a, supra)

(b) j'en connais des gens qui se sont retrouvés tout seuls dans les moments difficiles

+ et ben ça fait des dégâts quoi + ça fait des monstres dégâts $(=4 \mathrm{~h}$, supra $)$

Dans (16a), on peut comprendre que la quantité de fric était très importante; cette lecture quantitative est sans doute bien représentée dans le contexte 1, lorsque monstre est postposé (un banquet monstre, un breakfast monstre, TLFi) ${ }^{11}$. En revanche, en antéposition, les éventuelles lectures quantitatives apparaissent très exceptionnelles et nous paraissent liées au sémantisme de certains substantifs (comme fric). Ainsi pour (16b), faut-il comprendre que la solitude dans les moments difficiles entraîne de nombreux dégâts, une grande quantité de dégâts psychologiques, ou alors un degré élevé de dommages psychologiques ? À notre sens, la seconde lecture - intensive - est préférentielle.

Le contexte 4 est sans doute celui où la lecture quantitative est la mieux représentée : par exemple, dans il a monstre neigé, il est possiblement question de la quantité de neige : la lecture intensive n'est peut-être pas la seule envisageable. Dans il a monstre maillé sur lui, faut-il comprendre qu'il a râlé en quantité / à de nombreuses reprises ? ou alors qu'il l'a fait de manière disproportionnée ? Dans on a monstre discuté, est-il question de la quantité de mots, de discussions? ou alors de l'intensité des échanges ? Dans ces deux derniers exemples, la lecture quantitative - possible au demeurant - nous paraît plus improbable.

5.2 Le sémantisme de monstre épithète postposée : «exceptionnel par la quantité [...] l'intensité » (TLFi, supra, § 1.1.) explique qu'il puisse introduire une comparaison avec une intensité qui fait office de norme et placer le curseur largement au-delà de cette norme (Kleiber 2007). Porteur d'une composante subjective-évaluative, le marqueur monstre signifie ainsi une intensité extrême (parfois une quantité qui outrepasse les normes). 
Comment expliquer que monstre puisse se spécialiser comme intensifieur? Il n'est pas anodin d'antéposer un élément : «Synchroniquement, il est reconnu que le changement de position entraîne souvent un changement sémantique. En particulier, l'adjectif et l'adverbe expriment fréquemment un sens différent suivant leur position dans la phrase » (Shyldkrot 2007 : 44). La possibilité d'antéposition de l'épithète explique le sémantisme intensif de monstre, incident à un élément contigu. Fonctionnant comme marqueur de haut degré, monstre peut en quelque sorte « repasser à droite ", regagnant ainsi de nouveaux contextes (contextes 3 bis et 4 bis).

5.3 Comme d'autres marqueurs de haut degré, monstre présente un potentiel de renouvellement. Zribi-Hertz (2014:67) souligne que «les expressions à valeur intensive (haut degré) [...] sont [...] un observatoire privilégié de la créativité, du changement et de la variation linguistique (cf. Bolinger 1972) ». Bien qu'il marque le très haut degré, monstre peut lui-même être ré-intensifié, ce que l'on a montré avec le profil prosodique et des énoncés sur-intensifiés du type putain j'ai vraiment foutu une monstre merde (4f). Voyons les exemples (17):

(17) (a) y avait une monstre mais monstre descente! on est descendues sur les fesses (oral, à la volée, 15.09. 2019, canton de Fribourg; fille de 12 ans racontant une balade en famille dans la forêt)

(b) y avait un toboggan mais tu sais mais mon::stre long hein ! (oral, à la volée, canton de Fribourg ; fille de 11 ans, 07.08.2019)

(c) L1:-t'es déjà allé à Aigle à Parc Aventure ?

L2 : - ouais mais ça fait monstre monstre monstre longtemps! (oral, à la volée, canton de Fribourg ; la locutrice L2 est une fille de 11 ans, 07.08.2019)

(d) Mais c'est monstre trop bien ce truc! (web, https://monetoileinterieure.word press.com)

Dans (17a), on observe la réduplication de monstre de part et d'autre d'un mais « surréalisant » (García Negroni 1995, Richard 1999, Rodríguez Somolinos 2013) avec un effet de surenchère, doublé d'une proéminence sur la voyelle nasale (notée par les petites majuscules); dans (17b), la présence du mais «surréalisant» s'accompagne d'un allongement (noté par les deux points); dans (17c), la triplication de l'item est accompagnée d'une proéminence accentuelle sur la troisième occurrence ; dans (17d), l'intensif trop est cooccurrent à monstre.

\section{Conclusions}

Lorsqu'on se trouve confronté à des suites du type (18), la question se pose de la distinction entre le fonctionnement de monstre et celui de pire en Suisse romande - qui semblent fonctionner comme des parasynonymes :

(18) on est allé à la «grande Parade de Noël» dans les rues de Sydney !!! [...] Y'avait pire du monde et il faisait monstre chaud !! (web, https://funny-s-trip.skyrock.com, sic)

Le corpus OFROM ne fournit que trois occurrences de cet emploi (nos gloses entre parenthèses), quatre avec l'exemple (24) infra :

(19) (a) c'était le pire bazar quoi ('un bazar invraisemblable')

(b) et en fait je suis pas tombé amoureux tout de suite mais enfin j'ai déjà vu qu'elle était pire cool ('extrêmement cool')

(c) ce qui est pire cool c'est que j'ai pu choisir mon parrain de baptême 
Dans (19a), pire est épithète du N bazar dans le SN défini le pire bazar (contexte 1). Dans (19b-c), pire est incident à l'adjectif cool (contexte 3).

Le Corpus suisse de sms fournit 7 occurrences de pire :

(20) (a) Punaise ! Elle a fait un pire malaise ! ('un énorme / incroyable malaise')

(b) Mais arrete, chu sure k THE SHOW ca va etre 1pire truc,y zont mm un Trailer ! (sic, 'un truc de dingue')

(c) Moi chu à Neuch\&pire saoulée (sic, 'complètement soûle')

(d) c pire horrible! ('horribilissime')

(e) Elle a fait un truc pire moche ('un truc le plus moche qui soit')

(f) Trop pire forte la fille : 21 patients et pas de retard... La classe... ;-) ('extraordinairement forte')

(g) Hé vieux! On a pire cartoné !!! \o/ Hallucinant:-D (sic, 'cartonné comme jamais')

Tout comme pour (19), le contexte 2, où pire est épithète antéposée (20a-b), est attesté, mais le SN est ici indéfini. Le contexte 3 , du type pire cool, est illustré par (20c) à (20f). Le contexte 4 est également observable (20g). A priori, les contextes 3 bis et 4 bis ne sont pas possibles, pire étant «rarement postposé » (TLFi). Le contexte 5 apparaît également sporadiquement :

(21) A propos de la Grippe A, il y a pire beaucoup de cas au Japon! (web, https://nijiiro. skyrock.com)

Dans cet emploi, pire n'est donc pas orienté axiologiquement, contrairement à ses occurrences dans les contextes comparatifs. "Comparatif de supériorité de l'adjectif mauvais » (TLFi), pire est en effet utilisé, dans les emplois ci-dessus, sans orientation axiologique - tout comme monstre (cf. une monstre performance / une performance monstre brillante vs un monstre con / un mec monstre con). Par exemple dans le contexte 3 , elle était pire cool est fortement laudatif tout comme pire stylé (22a), alors que dans (22b-c) pire relou et un pire salaud sont fortement dépréciatifs :

(22) (a) elle est pire stylé comme tattooeuse! (web, www.carolinekarenine.com, sic)

(b) Le problème c'est que son coloc il devient vite bourré et il est pire relou. (web, www.jeuxvideo.com)

(c) putain j'suis un pire salaud des fois quand meme! (web, www.skipass.com)

Entrant dans la construction du superlatif, pire a tous les atouts pour fonctionner en tant que marqueur de haut degré. Les emplois du type le pire bazar, pire cool, on a pire cartonné ont en commun l'absence d'une composante comparative. Et pour cause, pire n'est pas comparatif, mais superlatif. Il signifie le plus haut degré qui soit, c'est-à-dire un degré par définition unique ${ }^{12}$.

Monstre et pire présentent d'indéniables affinités: contextes distributionnels en commun, sémantisme intrinsèquement intensif (superlatif avec le déterminant défini), portée de la négation (sont ainsi attestés à la fois c'est pire pas cool / monstre pas cool et c'est pas pire cool / c'est pas monstre cool (v. supra 7h-i)), etc.

Cela dit, pire et monstre présentent des différences quant à la position à droite de l'élément sur lequel porte le marqueur (pas de contextes 3 bis et 4 bis pour pire); par ailleurs, la valeur comparative de pire - bien qu'absente dans l'emploi qui nous intéresse constitue une différence avec monstre; enfin, si monstre fonctionne possiblement comme un indice de " parler jeune ", pire semble associé de manière plus évidente encore au parler des jeunes de Suisse romande. Les exemples (19, supra) sont produits par une femme de 32 ans, un homme étudiant et une femme de 22 ans. L'extrait (24), qui ressortit à la 
représentation personnelle de ses propres pratiques linguistiques, est énoncé par une femme de 20 ans :

(24) le langage jeune princip- je l'utilise quasiment que à l'oral + enfin après les expérien- des expressions tu sais enfin genre + mettre tout le temps des adjectifs genre c'était grave beau c'était euh: pire cool + ça oui à l'écrit aussi

D'autres questions restent en suspens qui devront faire l'objet d'une recherche future. D'une part, le lien éventuel de monstre épithète à monstrueux, et de monstre incident à un adjectif ou à un prédicat verbal à monstrueusement et monstrement (attesté par exemple dans les San Antonio). D'autre part, le rapport avec d'autres quantifieurs-intensifieurs, par exemple ben ben (Dostie 2018) ou grave (Zribi-Hertz 2014). Enfin, l'évaluation des hypothèses géo-historiques que conduit à formuler l'observation des emplois de monstre : le substrat francoprovençal et l'adstrat alémanique jouent-ils un rôle ? faut-il postuler un genre de renouvellement d'une forme ancienne (comme indiqué supra §1, le contexte 3 au moins - est répertorié depuis 1983)?

\section{Références}

Avanzi M. (2017). Atlas du français de nos régions. Paris : Armand Colin.

Avanzi M. (2018). «Le pronom $y$ accusatif en français régional et dans les dialectes gallo-romans : histoire et géographie ». Lingvisticae Investigationes 41/1, 62-86.

Avanzi, M. (2019). Parlez-vous les français ? Atlas des expressions de nos régions. Paris : Armand Colin.

Avanzi M., Béguelin M.-J. \& Diémoz F. (2016a). «Introduction », Corpus 15, 7-12.

Avanzi M., Béguelin M.-J. \& Diémoz F. (2016b). «De l'archive de parole au corpus de référence : la base de données orales du français de Suisse romande (OFROM) », Corpus 15, 309-342.

Avanzi M. \& Thibault A. (2018). "Réflexions épistémologiques sur de nouveaux apports méthodologiques et empiriques à l'étude géolinguistique des français d'Amérique ", Actes en ligne du CMLF. Mons (Belgique), juillet 2018. 〈https://doi.org/10.1051/shsconf/20184602001〉

Boersma P. \& Weenink D. (2019). "Praat: doing phonetics by computer ». Version 6.1.08, http://www.praat.org/

Corminboeuf G., Rothenbühler J. \& Sauzet M. (éds., à par.). Français parlés et français 'tout court', numéro thématique de la revue Studia Linguistica Romanica.

Dagnac A. \& Thuilier J. (à par.). «Français du Sud-Ouest et marquage différentiel de l'objet : une approche expérimentale ", L'Information Grammaticale 166.

Dostie G. (2018). Synonymie et marqueurs de haut degré. Sens conceptuel, sens associatif, polysémie. Paris : Classiques Garnier.

Fréchet Cl. (2015). Dictionnaire des régionalismes de Rhône-Alpes. Paris : Champion.

Gaatone D. (2008). «Un ensemble hétéroclite : les adverbes de degré en français ». Congrès Mondial de Linguistique Française - CMLF'08, 2495-2504.

Gaatone D. (2013). «Esquisse d'un guide des perplexes : problèmes de définition et de classification des adverbes de degré en français », Langue française 177, 37-50.

Gadet F. (2000). "Des corpus pour ne... pas», in: Bilger M. (éd.), Corpus : méthodologie et applications linguistiques. Paris : Champion, 156-167.

Gadet F. (2017). Les parlers jeunes dans l'Île-de-France multiculturelle. Paris/Gap : Ophrys.

García Negroni M. M. (1995). "Scalarité et réinterprétation: les modificateurs surréalisants », Anscombre J.-C. (éd.), Théorie des topoï. Paris : Kimé, 101-144.

Gratton Y. (2002). "Le krigeage. La méthode optimale d'interpolation spatiale ». Les articles de l'Institut d'Analyse Géographique, juin 2002, www.iag.asso.fr.

Kleiber G. (2007), "Adjectifs de couleur et gradation : une énigme... 'très' colorée », Travaux de linguistique 55, 9-44.

Kleiber G. (2013). « À la recherche de l'intensité », Langue française 177, 63-76.

Mahrer R. (2017). Phonographie. La représentation écrite de l'oral en français. Berlin : De Gruyter.

Martin J.-P. (1984). Description lexicale du français parlé en Vallée d'Aoste. Aoste : Musumeci Editeur.

Rézeau P. (2001). Le Dictionnaire des régionalismes de France. Bruxelles : De Boeck/Duculot. 
Richard E. (1999). "Félix est beau, mais beau!: du dit au dire», Revue de Sémantique et Pragmatique 5, 111-138.

$\mathrm{R}$ development core Team (2019). "R: A language and environment for statistical computing. $\mathrm{R}$ Foundation for Statistical Computing", Vienne (Autriche).

Rodríguez Somolinos A. (2013). "L'évolution de mais surréalisant en français : de fui t'en, mais vitement! à je kiffe, mais grave! ", Revue de sémantique et de pragmatique 33-34, 231-249.

Shyldkrot H. (2007). "Évaluation scalaire, identification et intensité : quand vrai n'est pas le contraire de faux », Travaux de linguistique 54, 43-56.

Thibault A. $\left(2004^{2}\right)$. Dictionnaire suisse romand. Carouge : Zoé.

Tuaillon G. \& Rézeau P. (1983). Document sur les français régionaux, ms., 143 p.

Zribi-Hertz A. (2015). "De l'évolution des propriétés du mot grave en français européen moderne », Dostie G. \& Hadermann P. (éds), La dia-variation en français actuel. Études sur corpus, approches croisées et ouvrages de référence. Berne : Peter Lang, 63-98.

Whittaker S. (2013). «L'intensification des noms de propriété », Langue française 177, 127-140.

\section{Ressources et corpus cités}

[BDLP, Base de données lexicographiques panfrancophone] Poirier C. (2005), «La dynamique du français à travers l'espace francophone à la lumière de la base de données lexicographiques panfrancophone », Revue de linguistique romane 69, 483-516. http://www.bdlp.org

[CFPQ, Corpus de français parlé au Québec] Dostie G. (2016), «Le Corpus de français parlé au Québec (CFPQ) et la langue des conversations familières : Exemple de mise à profit des données à partir d'un examen lexico-sémantique de la séquence je sais pas », Corpus 15, 115-133. https://recherche.flsh.usherbrooke.ca/cfpq/index.php/site/index

[ESLO, Enquêtes sociolinguistiques à Orléans] Baude O. \& Dugua C. (2016), «Les ESLO, du portrait sonore au paysage digital », Corpus 15, 29-56. http://eslo.huma-num.fr/

[Ofrom, Corpus oral de français de Suisse romande] Avanzi M., Béguelin M.-J. \& Diémoz F. (20122017), « Présentation du corpus OFROM - corpus oral de français de Suisse romande », Université de Neuchâtel. www.unine.ch/ofrom

[sms4science.ch, Corpus suisse de sms] Stark E., Ueberwasser S. \& Ruef B. (2009-2015). «Swiss SMS Corpus ». https://sms.linguistik.uzh.ch

[TLFi, Le Trésor de la langue française informatisé] ATILF-CNRS \& Université de Lorraine. www.atilf.fr/tlfi

[Français de nos régions] Avanzi M., Barbet C., Glikman J., \& Peuvergne J. (2016). « Présentation d'une enquête pour l'étude des régionalismes du français », Congrès Mondial de Linguistique Française-CMLF'16, non-paginé, https://francaisdenosregions.com

\footnotetext{
${ }^{1}$ Ces enquêtes visent à exploiter le potentiel des réseaux sociaux pour obtenir des données quant aux représentations de milliers d'internautes. Il s'agit des représentations, et non de ce qu'ils produisent réellement (Avanzi \& Thibault 2018).

${ }^{2}$ La forme gasconne hart peut être traduite en français par « rassasié »; elle existe aussi en emploi adverbial avec le sens de « assez » (FEW 3, 414b, FARCIRE II).

${ }^{3}$ Les cartes ont été générées avec différents 'packages' du logiciel R ( $\mathrm{R}$ development core Team, 2019)

${ }^{4}$ Pour des raisons d'espace, nous présentons directement la carte zoomée sur le Centre-Est (monstre n'est toujours pas employé ailleurs en France).

${ }^{5}$ Précisons que nous ne disons pas que monstre relève exclusivement des «parlers jeunes » (on est jeune seulement jusqu'à 20 , voire 25 ans...), mais que les participants qui disent l'utiliser sont en moyenne plus jeunes que ceux qui disent ne pas l'utiliser.

${ }^{6}$ Dans l'exemple (4k) le $\mathrm{N}$ est absent. On observe une interruption après tour, puis l'énonciation en parce que réalise un rattrapage d'information - afin sans doute de préciser qu'il ne s'agit pas d'une «tour » au sens où on l'entend en Europe. Une seconde interruption survient après l'épithète monstres, la locutrice reformulant monstres (tours) en gratte-ciels.

${ }^{7}$ Des occurrences recueillies à la volée confirment la vitalité de cet emploi :

L1 :- bien?
} 
L2 : - ah ouais:: monstre ! (oral, à la volée, 21.10.2018, canton de Vaud ; à propos d'un concert)

${ }^{8}$ Comme pour le contexte 3 , les exemples recueillis à la volée confirment l'existence de cet emploi :

(a) il m'a monstre énervé avec son 'mais t'inquiè::te' ! (oral, à la volée, 30.10.2019, canton de Fribourg; une adolescente dans le train)

(b) t'es monstre emmerdé si tu rates ta correspondance (oral, à la volée, 01.11.2019, canton de Fribourg ; étudiante à l'Université, à propos des retards des trains)

${ }^{9}$ Celles-ci ont été recueillies de manière non systématique, en raison de l'absence - dans les périodiques romands concernés - de ressources numériques interrogeables et en accès libre.

${ }^{10}$ L'intensif grave présente le même fonctionnement : le premier exemple illustre le contexte 3 bis, le second 4 bis et le troisième le contexte 5 . :

Et elle est belle grave, et en plus elle est dangereuse, croyez-moi. (web, https://alexandre2 pagesperso-orange.fr/brochette)

En direct de Zurich, Carlo aux platines et CL au micro qui déchirent leur race grave ! :) (sms, cité par Avanzi, Béguelin \& Diémoz 2016)

ton texte a fait grave beaucoup de vues !! c cool! (web, www.youtube.com)

${ }^{11}$ Mais rien n'est moins sûr. Un.e des relecteur/trice voit dans (16a) une intensification.

${ }^{12}$ Ainsi les exemples ci-dessous, qui contiennent un comparatif, se situent hors de notre champ (ils n'ont rien de « régional ») :

Son père était un pire salaud que ce qu'on pensait. (GoogleLivres, Hirsch, Comment j'ai tué son père)

Le tatoueur est le pire connard de cette île que je connaisse [...] (GoogleLivres, Romano, Endless Sommer) 